\title{
The design and synthesis of biodegradable pyridinium ionic liquids $\uparrow$
}

\author{
Jitendra R. Harjani, ${ }^{a}{ }^{\text {Robert D. Singer, }}{ }^{b}$ M. Teresa Garcia ${ }^{c}$ and Peter J. Scammells ${ }^{* a}$ \\ Received 11th January 2008, Accepted 15th February 2008 \\ First published as an Advance Article on the web 28th February 2008 \\ DOI: 10.1039/b800534f
}

Ionic liquids with a pyridinium cation bearing an ester side chain moiety were prepared from either pyridine or nicotinic acid and their biodegradability was evaluated using the $\mathrm{CO}_{2}$ Headspace test (ISO 14593). ILs of this type showed exceptionally high levels of biodegradation under aerobic conditions and can be classified as 'readily biodegradable'.

Ionic liquids (ILs) are deemed greener solvent alternatives in chemical synthesis mainly because of their negligible vapor pressure, high thermal stability, low flammability and reusability in chemical applications. ${ }^{1}$ They are also known to have an influence on the rate and selectivity of certain chemical reactions. Not surprisingly, with their numerous attributes these neoteric solvents have attracted the attention of chemists from a multitude of disciplines. In the last eight years, contributors from around the world have published more than 250 publications in this journal alone, mainly in the area of the synthesis of ILs or their use as green solvent alternatives. As research in the area of ILs continues to grow, the domain of their applications have substantially broadened. The non-volatility of ILs under operational conditions minimizes their impact on air quality during their life cycle. However, their impact on soil and water is certainly of considerable concern at the time of their disposal. Therefore, the quest to find a benign method of disposal for ILs is gaining momentum. ${ }^{2}$ The evaluation of the environmental impact of ILs can, in part, be gauged by parameters such as biodegradability and toxicity. ${ }^{2}$ Complete mineralization of any compound leads to non toxic $\mathrm{CO}_{2}$ and biomass, making its disposal a safe option. Designing biodegradable chemicals is significant in that it would decrease the bioaccumulation of the parent compound and its metabolic products, reducing its toxic effects. Our interest in ILs stems from their possible use in reactions that are important in organic synthesis. ${ }^{3}$ Designing benign chemicals is acknowledged as a crucial aspect of green chemistry., ${ }^{2,4}$ Hence, we believe that the logical design of ILs may lead to compounds that would not just be ideal solvents

${ }^{a}$ Medicinal Chemistry and Drug Action, Monash Institute of

Pharmaceutical Sciences, Monash University, Parkville, Victoria, 3052, Australia.E-mail: peter.scammells@vcp.monash.edu.au; Fax: +61 3 99039582; Tel: +61399039542

${ }^{b}$ Department of Chemistry, Saint Mary's University, Halifax, Nova Scotia, B3H 3C3, Canada

'Department of Surfactant Technology, IIQAB-CSIC, Jordi Girona 18-26, 08034, Spain. E-mail: mtgbet@iiqab.csic.es; Fax: +34 93204 5904; Tel: +34934006100

$\dagger$ Electronic supplementary information (ESI) available: Supplementary information on general preparation procedures. See DOI: 10.1039/ b800534f for chemical processes, but would also be safe for disposal and would therefore be sustainable. Research in this area is currently vital as ILs are likely to make a transition from academic laboratories to large scale operations where disposal of any chemical is a major concern. ${ }^{5}$ Enormous structural variations are possible in the ILs by changing either the cation and/or the anion. This leads us to believe that it should be possible to manipulate their chemical architecture to achieve high biodegradability.

Our initial work on the imidazolium based ILs has demonstrated that the introduction of the ester groups derived from a $\mathrm{C}_{2}$ acid and $\mathrm{C}_{4}$ or higher alcohol in the $3-N$-substitutent does increase their biodegradation. ${ }^{6}$ The $n$-octyl sulfate anion was integrated into the structure to meet the benchmark of 'readily biodegradable' materials. ${ }^{7}$ These observations clearly point toward the recalcitrance of the imidazolium core.

Although pyridinium based cationic surfactants are reported to have strong resistance towards biodegradation, ${ }^{8}$ some recent reports triggered our interest in them. This has lead to some noteworthy results which we disclose in this communication. The amphiphilic quaternary salts based on this moiety were identified to be potential candidates for gene delivery by Engberts and Hulst. ${ }^{9}$ In this report, the good biodegradability and low cytotoxicity of some compounds was claimed, but the quantitative assessment of the biodegradability was lacking. Kalka et al. examined some cationic surface active substances based on 1-dodecylpyridinium chloride. The study concluded that these compounds are generally toxic and only partly biodegradable. ${ }^{10}$ Very recently, Docherty et al. reported the mineralization of 1-hexyl-3-methylpyridinium bromide and 1octyl-3-methylpyridinium bromide after 49 and 25 days of incubation, respectively, by the DOC die-away test (OECD 301A). ${ }^{11}$

We focused our attention on the ILs that were based on the ester moiety as the introduction of this group in the imidazolium based ILs improved their biodegradation probably by providing a site for an enzymatic attack. ${ }^{6,7} \mathrm{The}^{\mathrm{CO}_{2}}$ headspace test (ISO 14593) ${ }^{7}$ was used to evaluate the biodegradability of the ionic liquids prepared in this study. If the biodegradation level measured according to this test is higher than $60 \%$ within twenty eight days, the compound can be considered as 'readily biodegradable'.

A simple quaternary bromide derived from pyridine and ethyl bromoacetate 1a and the corresponding octyl sulfate based IL 1b showed biodegradability at $87 \%$ and $89 \%$, respectively (Fig. 1). $\dagger$ Here the inclusion of the octyl sulfate anion resulted in no appreciable increase in biodegradation. This is unlike our earlier observation for the imidazolium based ILs, where the octyl sulfate anion significantly increased biodegradation. ${ }^{7}$ 


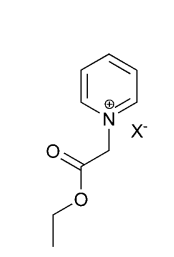

1a: $\mathrm{X}-=\mathrm{Br}^{-}$
1b: $\mathrm{X}-=\mathrm{C}_{8} \mathrm{H}_{17} \mathrm{OSO}_{3}$
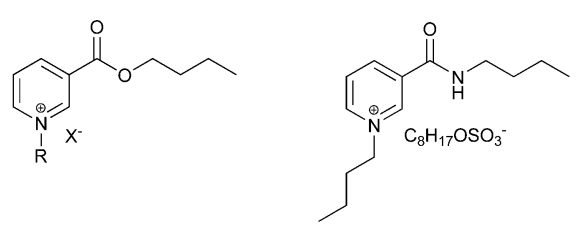

2a: $\mathrm{R}=\mathrm{CH}_{3}, \mathrm{X}-=\mathrm{I}$

2b: $\mathrm{R}=\mathrm{CH}_{3}, \mathrm{X}-=\mathrm{C}_{8} \mathrm{H}_{17} \mathrm{OSO}_{3}$

2c: $\mathrm{R}=\mathrm{C}_{4} \mathrm{H}_{9}, \mathrm{X}-=\mathrm{C}_{8} \mathrm{H}_{17} \mathrm{OSO}_{3}$. 2d: $\mathrm{R}=\mathrm{CH}_{3}, \mathrm{X}=\mathrm{NTf}_{2}^{-}$

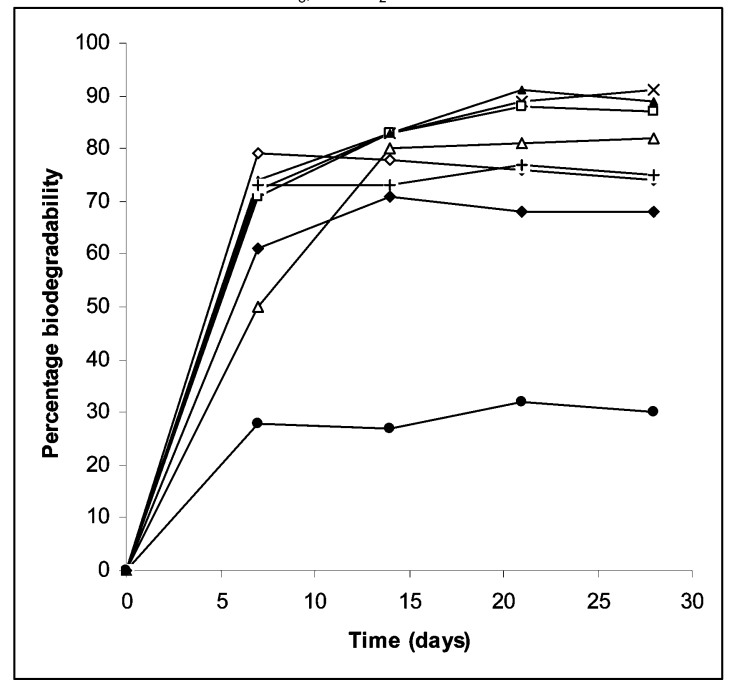

Fig. 1 Biodegradation of 1a $(\square), \mathbf{1 b}(\mathbf{\Delta}), \mathbf{2 a}(\diamond) \mathbf{2 b}(+), \mathbf{2 c}(\Delta), \mathbf{2 d}(\diamond)$, $3(\bullet)$ and sodium dodecyl sulfate (reference compound) $(\times)$ in the $\mathrm{CO}_{2}$ Headspace test.

Nicotinic acid, an inexpensive natural product based on pyridine, was an attractive starting material for the synthesis of pyridinium ionic liquids bearing functionality on the heterocyclic ring. Butyl nicotinate was readily obtained from nicotinic acid and was employed to derive some ILs. Its methylation with methyl iodide yielded $\mathbf{2 a}$; which upon treatment with sodium octyl sulfate yielded $\mathbf{2 b}$. Both $\mathbf{2 a}$ and $\mathbf{2 b}$ displayed biodegradation of $74 \%$ and $75 \%$, respectively (Fig. 1), once again demonstrating that the high biodegradability of this class of compounds is largely dependent upon the structure of the cation. A slight increase in the biodegradability from $75 \%$ to $82 \%$ with an increase in the alkyl chain length from $\mathrm{C}_{1}$ in $\mathbf{2 b}$ to $\mathrm{C}_{4}$ in $2 \mathbf{c}$ is perhaps due to an increase in lipophilicity. Although all the above levels of biodegradation are obtained after a standard 28 day period, it is notable that the biodegradability of $\mathbf{1 a}, \mathbf{1 b}, \mathbf{2 a}$ and $\mathbf{2 b}$ are so high that they degrade more than $70 \%$ within the first seven days of incubation. The percentages of mineralization attained under the stringent conditions of this biodegradation test allows these pyridinium compounds (1a, $\mathbf{1 b}, \mathbf{2 a}, \mathbf{2 b}$ and 2c) to be classified as readily biodegradable and therefore they would be expected to degrade rapidly and completely in aerobic aquatic environments.

Compounds 1a and $\mathbf{2 a}$ are solids at room temperature with melting points higher than $100{ }^{\circ} \mathrm{C}$; hence, these are not classified as typical ILs. However, the incorporation of a bis(triflimide) counterion afforded a compound (2d) which was both readily biodegradable and a liquid at room temperature.

Although the introduction of the amide group is known to improve the biodegradation of organic compounds, ${ }^{12}$ no signifi- cant improvement in the biodegradation was observed when this group was incorporated into the imidazolium based ILs. ${ }^{6}$ The nicotinamide based IL 3 was easily derived by quaternization of $N$-butyl nicotinamide with butyl iodide followed by the metathesis of the quaternary iodide using sodium octyl sulfate. The resultant IL 3 with two $\mathrm{C}_{4}$ linear aliphatic chains and an octyl sulfate anion showed poor biodegradation at 30\% after a 28 day period. Considering this as the contribution from the anion, we conclude that the presence of an amide group on the pyridinium ring does not improve biodegradation.

ILs $\mathbf{1 b}$ and $\mathbf{2 b}$ have comparable biodegradability to sodium dodecyl sulfate (SDS), a readily biodegradable anionic surfactant used as the reference compound in the biodegradation test. Intrigued by these preliminary results, we intend to continue exploring this class of IL further. Our main aim is to uncover the possible structural elements on the pyridinium core that are responsible for the higher biodegradation of this class of ILs. We are also interested in varying the anion relative to those commonly encountered in the ILs that would have an influence on the biodegradability. These studies can be useful in the design of ILs possessing good solvents attributes and high biodegradability. The details of this investigation will be disclosed in a subsequent full paper.

In conclusion, pyridine based ILs $\mathbf{1 b}$ and $\mathbf{2 b}$ and $\mathbf{2} \mathbf{c}$ showed strikingly high levels of biodegradability over the standard 28 day period. The high biodegradation rates appear to be a characteristic of the cation and do not depend on the counter ions tested. This presents the possibility that substituted pyridinium rings lead to metabolites that are not refractory upon biodegradation. The study also demonstrates that the structural manipulation of the pyridinium skeleton may lead to ILs which are likely to possess good solvent attributes and a predisposition to biodegrade when released into an aquatic environment.

\section{Acknowledgements}

The authors thank Pfizer Global R \& D, the Spanish Ministerio de Educación y Ciencia (CTQ2007-60364/PPQ) and the Australian Research Council (LX0561094) for financial support.

\section{Notes and References}

1 (a) J. Dupont, R. F. de Souza and P. A. Z. Suarez, Chem. Rev., 2002, 102, 3667; (b) J. S. Wilkes, Green Chem., 2002, 4, 73; (c) P. Wasserscheid and W. Keim, Angew. Chem., Int. Ed., 2000, 39, 3772; (d) T. Welton, Chem. Rev., 1999, 99, 2071.

2 (a) J. Ranke, S. Stolte, R. Störmann, J. Arning and B. Jastorff, Chem. Rev., 2007, 107, 2183; (b) R. S. Boethling, E. Sommer and D. DiFiore, Chem. Rev., 2007, 107, 2207.

3 S. Bouquillon, T. Courant, D. Dean, N. Gathergood, S. Morrissey, B. Pegot, P. J. Scammells and R. D. Singer, Aust. J. Chem., 2007, 60, 843.

4 (a) P. T. Anastas, J. C. Warner, Green Chemistry: Theory and Practice, Oxford University Press, Oxford, 1998; (b) Benign by Design, ed. P. T. Anastas and C. A. Farris, American Chemical Society, Washington, DC, 1994.

5 (a) D. Adam, Nature, 2000, 407, 938; (b) K. R. Seddon, Nat. Mater., 2003, 2, 363.

6 N. Gathergood, M. T. Garcia and P. J. Scammells, Green Chem., 2004, 6, 166.

7 N. Gathergood, P. J. Scammells and M. T. Garcia, Green Chem., 2006, 8, 156. 
8 P. H. Howard, in Handbook of Property Estimation Methods for Chemicals: Environmental and Health Science, Lewis Publishers, Boca Raton, FL, 2000, ch. 12, pp. 281-310.

9 (a) A. Roosjen, J. Šmisterová, C. Driessen, J. T. Anders, A. Wagenaar, D. Hoekstra, R. Hulst and J. B. F. N. Engberts, Eur. J. Org. Chem., 2002, 1271; (b) D. Pijper, E. Bulten, J. Šmisterová, A. Wagenaar, D. Hoekstra, J. B. F. N. Engberts and R. Hulst, Eur. J. Org. Chem., 2003, 4406.
10 E. Grabińska-Sota and J. Kalka, Environ. Int., 2003, 28, 687.

11 K. M. Docherty, J. K. Dixon and C. F. Kulpa Jr., Biodegradation, 2007, 18, 481.

12 (a) R. S. Boethling, Designing Safer Chemicals, ACS Symp. Ser. 640, 1996, 156; (b) P. H. Howard, R. S. Boethling, W. Stiteler, W. Meylan, J. Beauman, Sci. Total Environ., 1991,110/635; (c) R. S. Boethling, Cationic Surfactants, Surfactant Science Series Vol. 53, Marcel Dekker, New York, 1994, pp. 95-135. 